\title{
Periodic entrainment of power dropouts in mutually coupled semiconductor lasers
}

\author{
J.M. Buldú \\ Departament de Física i Enginyeria Nuclear, Universitat Politècnica de Catalunya, Colom 11, E-08222 Terrassa, Spain \\ Raúl Vicente, Toni Pérez, and Claudio R. Mirasso \\ Departament de Física, Universitat de les Illes Balears, E-07071 Palma de Mallorca, Spain \\ M.C. Torrent and J. García-Ojalvo \\ Departament de Física i Enginyeria Nuclear, Universitat Politècnica de Catalunya, Colom 11, E-08222 Terrassa, Spain
}

(Dated: August 14, 2002)

\begin{abstract}
We examine the effect of current modulation in the irregular dropout dynamics exhibited by two mutually coupled semiconductor lasers. Our experimental results show that a weak periodic modulation in the injection current of one of the lasers entrains the power dropouts in a very efficient way. It is also observed that the laser with the highest frequency leads the dynamics independently of which laser is modulated. As a result, the entrainment is anticipative when modulation is applied to the laser with lowest frequency. Numerical simulations of a model based on delay-coupled rate equations successfully reproduce the observed behavior.
\end{abstract}

PACS numbers: 05.40.-a, 42.65.Sf, 42.55.Px

Synchronization of coupled lasers has emerged in recent years as the basic mechanism underlying applications such diverse as high-power coherent emission by laser arrays [1], quantum-noise reduction via twin-beam generation [2], and information transmission in chaotic communication systems [3]. In the latter context, information is encoded in the chaotic carrier generated by an emitter laser, and decoded by a receiver laser to which the emitter is synchronized. Most of the schemes designed to that end are based on unidirectional coupling, in which the light emitted by one laser is partially injected into the other laser. However, certain attention has also been directed to the case of bidirectional coupling, in which the two lasers equally affect one another through mutual injection [4]. Recent investigations of this scheme have shown that mutual coupling destabilizes the otherwise steady-state operation of the lasers, by inducing sudden power dropouts occurring irregularly during the time evolution of the synchronized lasers, at frequencies of the order of megahertz $[5,6]$. The mechanism leading to this instability is believed to be similar to that involved in the occurrence of low frequency fluctuations in semiconductor lasers subject to optical feedback [7]. In the present paper we show that the irregular power dropouts exhibited by two mutually coupled lasers can be entrained periodically in a very efficient way, by adding a small amplitude modulation to the injection current of one of the lasers.

Our experimental setup is shown schematically in Fig. 1. We use two index-guided AlGaInP semiconductor lasers (Roithner RLT6505G) with a nominal wavelength of $650 \mathrm{~nm}$, whose injection current (temperature) is controlled with an accuracy of $\pm 0.1 \mathrm{~mA}\left( \pm 0.01^{\circ} \mathrm{C}\right)$. In the results presented here, we set the temperatures of the lasers to $T_{1}=18.15^{\circ} \mathrm{C}$ and $T_{2}=22.25^{\circ} \mathrm{C}$, for which the solitary laser thresholds are $I_{1}^{\text {th }}=17.5 \mathrm{~mA}$ and $I_{2}^{\text {th }}=17.3 \mathrm{~mA}$, respectively. The output of each laser is collimated by an antireflection-coated laser-diode objective, and injected into the other laser at a distance of $1.02 \mathrm{~m}$, which corresponds to an external cavity of $\tau_{c}=3.4 \mathrm{~ns}$. The threshold reduction due to the feedback introduced by the facet of the opposite laser is $1.71 \%$ in laser 1 and $1.16 \%$ in laser 2 . We note that these feedback strengths are not large enough to introduce any significant dynamical behavior in each laser when the other one is turned off. A sinusoidal modulation is introduced in one of the lasers through an Agilent 33120A function generator.

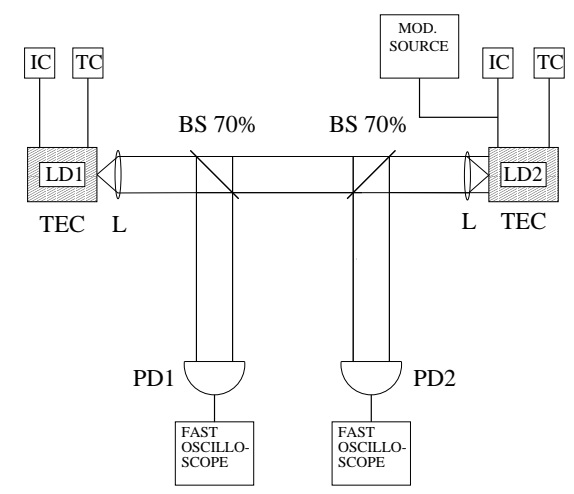

FIG. 1: Experimental setup: LD, laser diode; BS, beam splitter; TEC, laser diode mount; PD, photodiode; IC, injection current source; TC, temperature controller.

In order to maximize the interaction between the mutually coupled lasers, we force them to operate at wavelengths as similar as possible by adjusting their input currents. For $I_{1}=17.8 \mathrm{~mA}$ and $I_{2}=17.7 \mathrm{~mA}$ the lasers have a similar optical spectrum, centered at $\lambda=$ $657.0 \mathrm{~nm}$. Under these conditions, the output intensities of both lasers exhibit synchronized power dropouts, as shown in Fig. 2(a), which displays the intensity evolu- 
tion of one of the lasers (the other one is basically identical). These dropouts are irregularly spaced in time, as can be seen in the probability distribution function of the time interval between consecutive dropouts, displayed in Fig. 2(b). When a $10 \mathrm{MHz}$ sinusoidal modulation is added to the injection current of one of the lasers, the dropouts start to become entrained to the external periodic driving. For low modulation amplitudes the intensity dropouts occur at multiples of the modulation period [Fig. 2(c,d)], and if the amplitude is further increased we can finally observe a complete entrainment to the modulation period [Fig. 2(e,f)]. We note that the levels of modulation amplitude required to reach this entrainment are low, in comparison to the mean bias level of the injection current $(\sim 1.7 \%)$. This contrasts, for instance, with the case of the dropouts exhibited by a single laser subject to optical feedback, for which the modulation required to get entrainment is so large that it substantially distorts the overall dynamics of the laser (see for instance Fig. 3 in Ref. [11]).

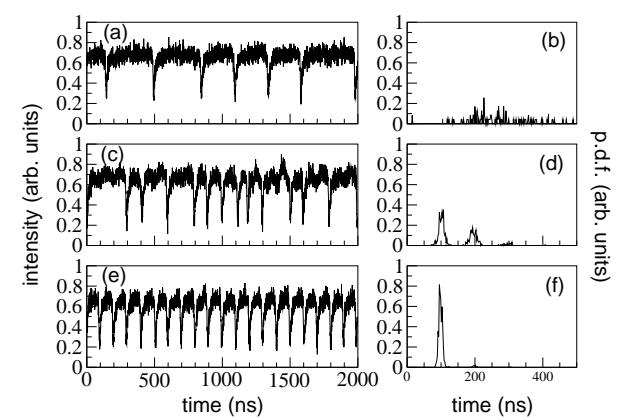

FIG. 2: Time evolution and the corresponding probability distribution function of the intensity dropouts, for increasing values of modulation amplitude: $0 \mathrm{~mA}(\mathrm{a}, \mathrm{b}), 0.23 \mathrm{~mA}(\mathrm{c}, \mathrm{d})$ and $0.30 \mathrm{~mA}(\mathrm{e}, \mathrm{f})$ respectively.

Both with and without modulation, the dropouts of the two lasers are synchronized with a constant delay time approximately equal to the flight time between the lasers $\pm \tau_{c}$. For a nonzero detuning (but small enough to maintain synchronization), the laser with higher frequency always leads the dynamics [5], an effect which can be related to the asymmetric response of semiconductor lasers to injection. Figure $3(\mathrm{a}, \mathrm{b})$ shows the intensity time traces of both lasers in the case of complete entrainment, when the leader laser is modulated. It can be seen that the dropouts occur earlier in the leader than in the laggard laser. In order to quantify this fact, Fig. 3(c) shows a synchronization plot of the two time series, with the intensity of the laggard laser advanced $\tau_{c}=3.4 \mathrm{~ns}$.

A previous analysis of the chaos pass filtering properties of two mutually coupled lasers has shown that the leader and laggard roles are clearly different, with the leader synchronizing the laggard but not the other way around [5]. However, we have observed that modulating the laggard instead of the leader does not affect the order of the dropouts. Therefore, the entrainment

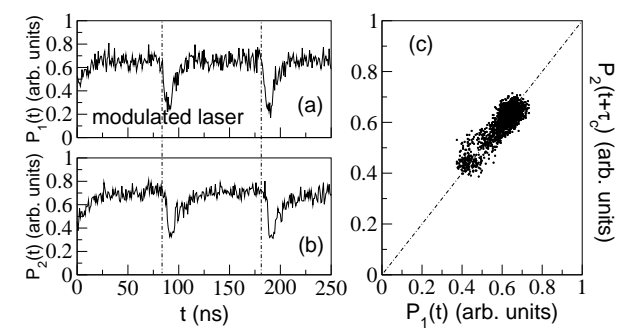

FIG. 3: Intensity time traces of the two lasers (a, b) and delayed synchronization plot (c), in the situation of complete entrainment and when the laser with higher frequency is modulated.

is transferred from the laggard to the leader in a form of anticipated synchronization [8-10]. This situation is displayed in Fig. 4, which shows how laser 1 (the one with higher frequency), even though it is not modulated, exhibits dropouts at the modulation period anticipating the behavior of the modulated laser. We note that we observe a symmetric scenario by changing the sign of the detuning between the two lasers (thus ruling out any systematic effect due to qualitative differences between them).

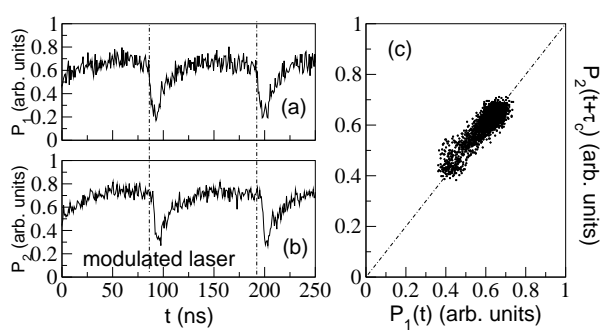

FIG. 4: As in Fig. 3, but with the modulation being applied to the laser with lower frequency.

With the aim of reproducing the experimental observations, we have studied a phenomenological model which describes the behavior of the system by means of rate equations for the complex slowly-varying electrical fields $E_{1,2}$ and carriers $N_{1,2}$ of the two lasers [12]:

$$
\begin{gathered}
\frac{d E_{1,2}}{d t}=\frac{(1+i \alpha)}{2}\left[G_{1,2}-\gamma\right] E_{1,2} \pm i \Delta \omega E_{1,2} \\
+\kappa e^{-i \Omega \tau_{c}} E_{2,1}\left(t-\tau_{c}\right)
\end{gathered}
$$

where $G_{1,2}(t)=\left[g\left(N_{1,2}-N_{0}\right)\right] /\left[1+s P_{1,2}(t)\right]$ and the electric fields rotate at a symmetric reference frequency $\Omega=\left(\omega_{1}+\omega_{2}\right) / 2$, with $\omega_{1,2}$ representing the free-running optical frequencies of the two lasers. The last term in Eq. (1) accounts for the delayed injection between the lasers. The optical intensity (or number of photons inside the cavity) is given by $P_{1,2}(t)=\left|E_{1,2}(t)\right|^{2}$. We assume that the two lasers have identical linewidth enhancement factor $\alpha=3.5$, differential gain $g=1.2 \times 10^{-8} \mathrm{ps}^{-1}$, 
gain saturation factor $s=5 \times 10^{-7}$, and carrier number at transparency $N_{0}=1.25 \times 10^{8}$. Other parameters are assumed to differ slightly between the lasers, namely the cavity losses $\gamma_{1}=0.687 \mathrm{ps}^{-1}$ and $\gamma_{2}=0.496 \mathrm{ps}^{-1}$, and the carrier decay rate $\gamma_{e 1}=0.601 \mathrm{~ns}^{-1}$ and $\gamma_{e 2}=$ $0.651 \mathrm{~ns}^{-1}$. These values have been chosen in order to provide conditions similar to the experimental ones, reproducing the threshold currents $\left(I_{t h 1}=17.3 \mathrm{~mA}\right.$ and $I_{t h 2}=17.5 \mathrm{~mA}$ ). The time delay is $\tau_{c}=3.4 \mathrm{~ns}$, while $\kappa$ is fitted to $20 \mathrm{~ns}^{-1}$. The leader-laggard dynamics is obtained by introducing a detuning between the laser frequencies $\Delta \omega=\left(\omega_{1}-\omega_{2}\right) / 2=2 \mathrm{GHz}$.

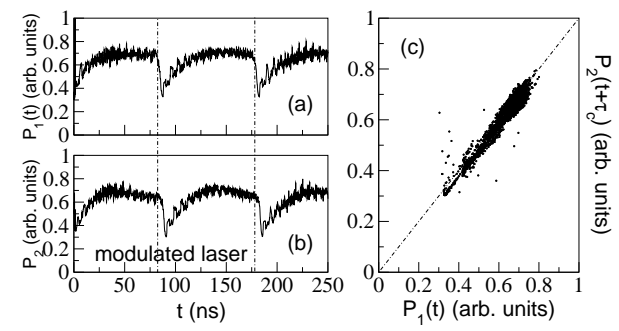

FIG. 5: Numerical results corresponding to the experimental time series of Fig. 4. The time series have been filtered at $400 \mathrm{MHz}$, in order to reproduce the bandwidth of the experimental detectors.

In the presence of harmonic driving, the injection current takes the form $I_{1,2}=I_{b 1, b 2}+A_{1,2} \sin \left(2 \pi t / T_{m}\right)$, where
$A_{1,2}$ is the modulation amplitude and $T_{m}$ is its period. In the numerical simulations, $I_{b 1, b 2}$ are adjusted to the respective threshold currents and $T_{m}$ is $100 \mathrm{~ns}$. The numerical results are in perfect agreement with the experimental data, and the entrainment of the power dropouts is already observed when both the leader and the laggard are modulated with relatively small modulation amplitude. As an example, we show in Fig. 5 numerical results for $A_{1,2}=0.3 \mathrm{~mA}$, exhibiting entrained dropouts when the laggard laser (the one with lower frequency) is modulated. Clearly, anticipated entrainment and a high degree of correlation is observed (upon advancing $\tau$ the modulated time series), in very good agreement with the experiment. The same kind of entrainment, but retarded, is observed when modulating the leader laser.

In conclusion, two mutually coupled semiconductor lasers have been experimentally and numerically analyzed when the injection current of one of the lasers is subject to harmonic modulation. Entrainment of the coupling-induced power dropouts at the modulation period is obtained for relatively low modulation amplitudes, independently of whether the leader or the laggard laser is modulated. Therefore, anticipative entrainment is observed when the laggard laser is modulated.

We acknowledge financial support from MCyT (Spain, projects BFM2001-2159 and BFM2002-04369), from the EU IST network OCCULT, and from the Generalitat de Catalunya (project 2001SGR00223).
[1] J.R. Leger, M.L. Scott, and W.B. Veldkamp, Appl. Phys. Lett. 52, 1771 (1988); J. García-Ojalvo, J. Casademont, C.R. Mirasso, M.C. Torrent, and J.M. Sancho. Int. J. Bif. Chaos 9, 2225 (1999)

[2] C. Serrat, M. C. Torrent, J. Garcia-Ojalvo, and R. Vilaseca, Phys. Rev. A 64, 041802(R) (2001); C. Serrat, R. Vilaseca, G. Bouwmans, B. Ségard, and P. Glorieux, Phys. Rev. A, to be published (2002).

[3] P. Colet and R. Roy, Opt. Lett. 19, 2056 (1994); C.R Mirasso, P. Colet, and P. García-Fernández, IEEE Phot. Technol. Lett. 8, 299 (1996); G.D. VanWiggeren and R. Roy, Science 279, 1198 (1998); J.P. Goedgebuer, L. Larger, and H. Porte, Phys. Rev. Lett. 80, 2249 (1998); S. Sivaprakasam and K.A. Shore, Opt. Lett. 24, 1200 (1999); I. Fischer, Y. Liu, and P. Davis, Phys. Rev. A 62, 011801(R) (2000); H.D.I. Abarbanel, M.B. Kennel, L. Illing, S. Tang, H.F. Chen, and J.M. Liu, IEEE J. Quantum Electron. 37, 1301 (2001).
[4] A. Hohl, A. Gavrielides, T. Erneux, and V. Kovanis, Phys. Rev. Lett. 78, 4745 (1997).

[5] T. Heil, I. Fischer, W. Elsäßer, J. Mulet, and C.R. Mirasso, Phys. Rev. Lett. 86, 795 (2001).

[6] C.R. Mirasso, M. Kolesik, M. Matus, J.K. White, and J.V. Moloney, Phys. Rev. A 65, 013805 (2002).

[7] G.H.M. van Tartwijk and G.P. Agrawal, Prog. Quantum Electron. 22, 43 (1998).

[8] Y. Liu, Y. Takiguchi, P. Davis, T. Aida, S. Saito, and J.M. Liu, Appl. Phys. Lett. 80, 4306 (2002).

[9] S. Sivaprakasam, E.M. Shahverdiev, P.S. Spencer, and K.A. Shore, Phys. Rev. Lett. 87154101 (2001).

[10] C. Masoller, Phys. Rev. Lett. 86, 2782 (2001).

[11] D.W. Sukow and D.J. Gauthier, IEEE J. Quantum Electron. 36, 175 (2000).

[12] J. Mulet, C. Masoller, and C.R. Mirasso, Phys. Rev. A 65, 063815 (2002). 